\title{
NEW EVIDENCE FOR ACCRETION DISKS IN AGNs
}

\author{
Jules P. Halpern and Kaiyou Chen \\ Columbia Astrophysics Laboratory \\ Columbia University \\ 538 W. 120th Street \\ New York, New York 10027
}

\begin{abstract}
We have refined our calculation of the line profile of a relativistic, Keplerian disk by incorporating a variety of emissivity laws, as well as broadening due to turbulence or electron scattering. The significant improvement in the fit to the double-peaked $\mathrm{H} \alpha$ line profile of the elliptical radio galaxy Arp 102B provides the most convincing direct evidence for an accretion disk in any AGN. Arp 102B appears to be a low-luminosity analog of 3C 390.3 , and several lines of evidence point to the existence of small, hot ion tori illuminating an outer thin disk in both of these galaxies. The rarity of these emission-line profiles might be understood if this particular combination of ion torus/thin disk occurs only for a narrow range of $\dot{m}\left(=\dot{M} / \dot{M}_{\text {Edd }}\right)$.
\end{abstract}

\section{INTRODUCTION}

Only a handful of AGNs have the double-peaked, broad emission lines which are characteristic of a rotating disk, but these rare examples offer the most direct evidence for the reality of accretion disks around supermassive black holes. The prime candidates are radio galaxies such as 3C 390.3 (Oke 1987; Perez et al. 1988) and Arp 102B (Halpern and Filippenko 1988; Chen, Halpern, and Filippenko 1988). Also, the variable Balmer lines of the Seyfert galaxy Akn 120 sometimes appear double peaked (Peterson et al. 1985), and an interpretation in terms of an accretion disk would require the component contributed by the disk to be highly variable (Alloin, Boisson, and Pelat 1988). The high quality of the spectroscopic data being obtained demands a rigorous general relativistic treatment of the line profile expected from a Keplerian disk. This was done by Chen, Halpern, and Filippenko (1988), who demonstrated that the effects of Doppler boosting and gravitational redshift are not small perturbutions to the non-relativistic case, but are essential to the understanding of the line profile arising in a disk. The blueshifted peak is always higher than the redshifted one, and there is a net gravitational redshift of the entire line. These effects enable one to fit for the inclination angle of the disk, a quantity which is not uniquely measured in non-relativistic Keplerian motion.

In this paper, we describe refinements to the model of Chen, Halpern, and Filippenko (1988, hereafter CHF) which result in a major improvement in the agreement with the data on Arp 102B. In particular, we find evidence for broadening of the line by electron scattering locally in the disk, where the electron temperature is less than $10^{5} \mathrm{~K}$. We also propose a physical model in which a small ion-supported torus (Rees et al. 1982; Begelman 1985) occupies the inner disk, and illuminates the thin outer disk with ionizing radiation. 


\section{LINE PROFILE OF A KEPLERIAN DISK}

\subsection{Simple Power-Law Emissivity}

CHF calculated the line profile of a Keplerian disk in the weak-field limit. The specific intensity of a line of frequency $\nu_{o}$ was represented by a delta function in the rest frame of the emitting particle, with the emissivity $\epsilon$ varying as a power $q$ of the dimensionless radius $\xi=c^{2} r / G M$ between inner and outer radii $\xi_{1}$ and $\xi_{2}$. Thus,

$$
\epsilon(\xi)=\frac{\epsilon_{o}}{4 \pi} \xi^{-q} \quad \operatorname{erg} \mathrm{cm}^{-2} \mathrm{~s}^{-1} \mathrm{sr}^{-1}
$$

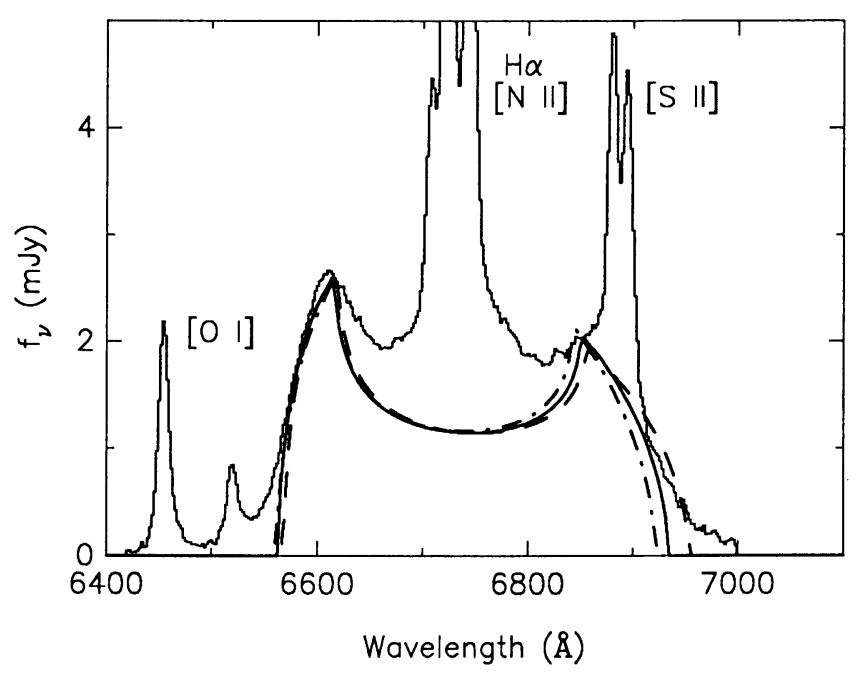

Figure 1. Fit of simple power-law emissivity to the $\mathrm{H} \alpha$ line of Arp 102B. Solid line corresponds to $i=33^{\circ} .5, \xi_{1}=400, \xi_{2}=1000, q=3.1$; dashed line to $i=28^{\circ}, \xi_{1}=270, \xi_{2}=$ $720, q=3.2$; dot-dashed line to $i=39^{\circ}, \xi_{1}=550, \xi_{2}=1400, q=3.0$.

and $I_{\nu e}=\epsilon(\xi) \delta\left(\nu_{e}-\nu_{o}\right)$. The observed line profile was then calculated using the invariance of the quantity $I_{\nu} / \nu^{3}$. When fitting to an observed line, the parameters $\xi_{1}, \xi_{2}$, and $q$ can, in principle, be determined by the shape of the line, as can the inclination angle $i$. The normalization fixes the product $\epsilon_{o} M^{2}$.

Figure 1 shows a range of fits which can reasonably be accommodated by the broad $\mathrm{H} \alpha$ profile of Arp 102B. The disk models do not account for all of the $\mathrm{H} \alpha$ flux, especially near zero velocity. Therefore, we have taken the approach that the disk-like component should be fitted to as much of the flux as possible without exceeding the observation at any point. The limiting factor in the accuracy of the fit is the uncertainty in the amount of flux to attribute to the [S II] lines. The best fit corresponds to the parameters $i=33^{\circ} .5, \xi_{1}=400, \xi_{2}=1000$, and $q=3.1$. The inclination angle can be determined to $\pm 5^{\circ} .5$ because the effects of gravitational and transverse redshift break the degeneracy with respect to inclination angle and radius in Keplerian motion. The emissivity index $q$ is determined by the steepness of the blue side of the profile, and the error is about \pm 0.3 . 


\subsection{A Continuous Emissivity Law}

The fit to the line can be greatly improved by the use of an emissivity law which does not have sharp inner and outer boundaries. If we allow the emissivity first to increase with radius, then to decrease smoothly, a better match to the wings as well as the peaks is achieved. We have chosen to parameterize the emissivity as follows:

$$
\epsilon(\xi) \propto \begin{cases}\left(\xi / \xi_{1}\right)^{q} & \xi<\xi_{1} \\ \left(\xi / \xi_{1}\right)^{-q} e^{-\left(\xi-\xi_{1}\right)^{2} / \xi_{2}^{2}} & \xi \geq \xi_{1}\end{cases}
$$

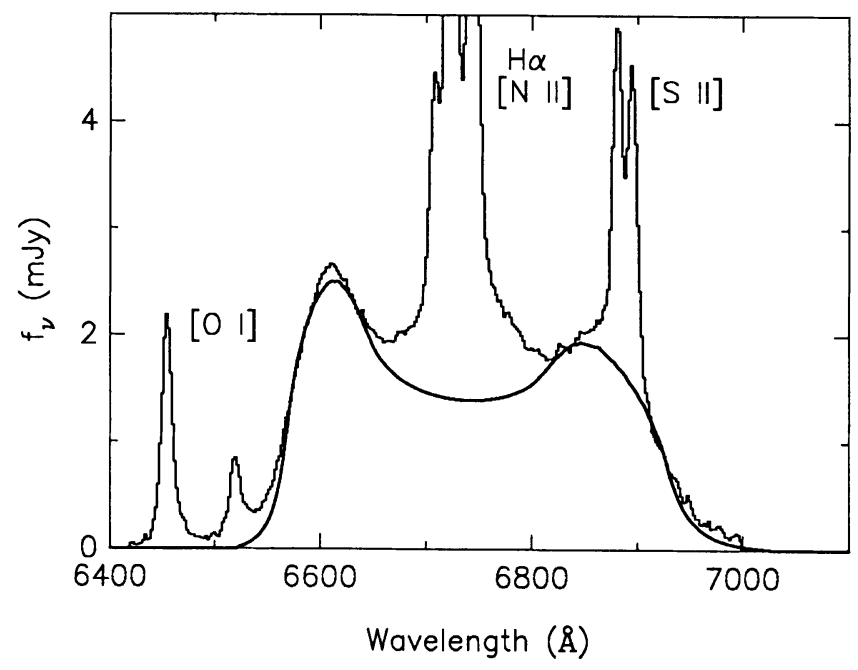

Figure 2. Fit of continuous emissivity law to the $\mathrm{H} \alpha$ line of Arp 102B. Parameters are $i=32^{\circ}, \xi_{1}=400, \xi_{2}=900$, and $q=3.0$.

Note that this continuous emissivity law, although ad hoc, has the same number of free parameters as the simple power-law model. Figure 2 shows the best fit which we have achieved, with $i=32^{\circ}, \xi_{1}=400, \xi_{2}=900$, and $q=3.0$. The parameters are virtually unchanged from the simple power-law model, but now the added emissivity at $\xi<\xi_{1}$ contributes to the line wings, while the gradually changing slope due to the exponential term broadens the two peaks. Other emissivity laws have been explored, and it is generally found that the emissivity peaks at about $\xi=400$, and that $q$ and $i$ are restricted to values near 3.0 and $33^{\circ}$, respectively. We also note that no disk model can account for all the broad-line emission. After subtraction of the model from the data, the $\mathrm{H} \alpha$ line still has broad wings centered at zero velocity, similar to those in a Seyfert 1.5 galaxy. Thus, Arp 102B probably has "standard" broad-line clouds in addition to the disk component.

\subsection{Broadening by Electron Scattering or Turbulence}

The final emissivity law is qualitatively different from the first two in that it simulates local broadening of the line by replacing the delta function in the specific intensity by a Gaussian 
function of frequency. In this case, we retain the simple power-law emissivity of Equation (1), so that the specific intensity becomes

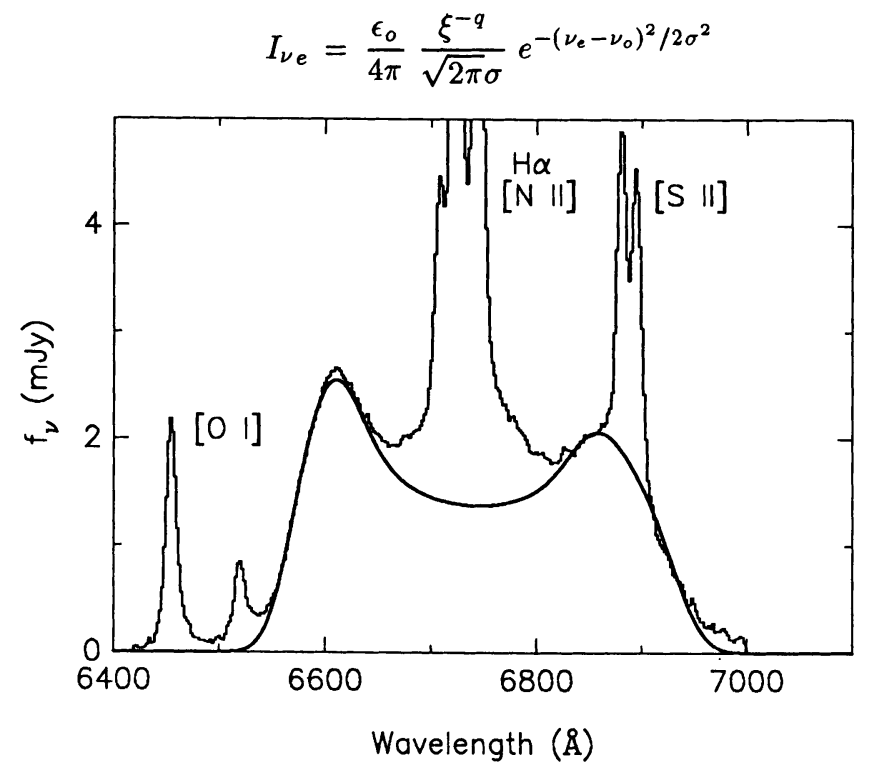

Figure 3. Fit of line-broadened emissivity law to Arp 102B. Parameters are $i=32^{\circ}, \xi_{1}=$ $350, \xi_{2}=1000, c \sigma / \nu_{o}=850 \mathrm{~km} \mathrm{~s}^{-1}$.

for $\xi_{1}<\xi<\xi_{2}$. At the expense of the addition of one more free parameter, $\sigma$, this model provides a better fit than any other which we have tried. Figure 3 shows the fit obtained for $i=32^{\circ}, \xi_{1}=350, \xi_{2}=1000, q=3.0$, and $\sigma / \nu_{o}=2.8 \times 10^{-3}$. This value of $\sigma / \nu_{o}$ corresponds to a velocity dispersion of $850 \mathrm{~km} \mathrm{~s}^{-1}$, or a Gaussian FWHM of $2000 \mathrm{~km} \mathrm{~s}^{-1}$. Actually, the velocity dispersion derived in this fit should be considered an upper limit to the true value, since some fraction of the broadening may be contributed by a continuous change in the emissivity index $q$ as described in the previous section.

There are several possible physical interpretations of the line broadening. Doppler broadening due to thermal motions of the emitting atoms seems highly unlikely, since the equivalent temperature is $\sim 10^{8} \mathrm{~K}$. Similarily, turbulence of this velocity would be highly supersonic in $\mathrm{a} \sim 10^{4} \mathrm{~K}$ gas which is necessary for Balmer emission, and would probably be dissipated in shocks. A very attractive possibility is that we are seeing the effects of electron scattering in a photoionized atmosphere of the disk. Shields and McKee (1981) showed that the FWHM of a scattered line is approximately $2000 T_{5}^{1 / 2}\left(1+\left\langle n_{s}\right\rangle\right)^{1 / 2} \mathrm{~km} \mathrm{~s}^{-1}$, where $T_{5}$ is the temperature in units of $10^{5} \mathrm{~K}$ and $\left\langle n_{s}\right\rangle$ is the mean number of scatterings. Our measured FWHM implies an upper limit to the temperature, although the scattering profile is not not exactly a Gaussian. Since there is no obvious unscattered core to the line, it is likely that $\left\langle n_{s}\right\rangle$ is substantially larger than 1 . For example, it may be that $1+\left\langle n_{s}\right\rangle \simeq 5$, so that $T \simeq 20,000 \mathrm{~K}$, typical of photoionized, broad-line regions. 


\section{PHYSICAL INTERPRETATION}

CHF showed that the peak $\mathrm{H} \alpha$ flux from the disk in Arp 102B is $\simeq 5 \times 10^{9} M_{8}^{-2} \mathrm{erg} \mathrm{cm}^{-2}$ $\mathrm{s}^{-1}$, which is within a factor of 2 of the energy flux from a standard thin accretion disk (Shakura and Sunyaev 1973) at $\xi \simeq 400$. For this reason, a local source of energy for the lines is possible only if virtually all the available energy is converted into line emission. More likely, photoionization of the surface of the disk by radiation from the inner disk or central nonthermal source is needed. This requirement was also deduced by Collin-Souffrin (1987) for the line emssion from AGN disks in general. CHF also estimated that the effective temperature of a standard $\alpha$-disk would be $\leq 5000 \mathrm{~K}$ at the radius of the $\mathrm{H} \alpha$ emission in Arp $102 \mathrm{~B}$, so that an additional source of heating is required to account for the recombination Balmer line ratios. The fit to $q \simeq 3$ is consistent with an isotropic, spherical source of illumination, concentric with a thin disk, providing that the disk is much larger than the source (Mardaljevic, Raine, and Walsh 1988). So in addition to solving the energy budget problem and heating the electrons to broaden the line profiles, a central photoionizing source also accounts for the observed value of the emissivity index $q$.

Another unusual property of Arp 102B, which is shared by 3C 390.3 as well, is a peak in the far-infrared (IRAS) spectrum at $25 \mu \mathrm{m}$. Most AGN spectra turn over at longer wavelengths, if at all. Although Miley et al. (1984) concluded that the peak in 3C 390.3 is probably due to dust, we point out that exactly such a feature was predicted to exist in the nonthermal spectra of radio galaxies by Rees et al. (1982) prior to the IRAS observations. The ion-supported torus is a theoretical construct which was proposed to explain the jets in radio galaxies, which apparently have a low accretion luminosity. Because of the small $\dot{m}$, the electrons can decouple from the ions inside a certain radius, where $T_{i}>T_{e}$ and the disk can no longer radiate efficiently. The torus collimates the radio jets, and the primary source of radiation is electron synchrotron in the far infrared, calculated assuming an equipartition magnetic field. The key prediction, which may now have been observationally verified in $3 \mathrm{C} 390.3$ and Arp 102B, is that the synchrotron self-absorption frequency is $\sim 10^{13} \mathrm{~Hz}$, or $\sim 30 \mu \mathrm{m}$ (Rees et al. 1982; Begelman 1985, 1988). Possibly, the observed peak is due to this turnover. The tendency of AGNs in general to have strong $25 \mu \mathrm{m}$ flux relative to 60 and $100 \mu \mathrm{m}$ may mean that a self-absorption frequency near $\sim 10^{13} \mathrm{~Hz}$ is common, while most of the 60 and $100 \mu \mathrm{m}$ flux is extranuclear.

We propose a model for both 3C 390.3 and Arp 102B in which a quasi-spherical, hot ion torus occupies the inner disk $(\xi<100)$, and illuminates the thin outer disk. The ionizing radiation could consist of Compton scattered photons from the ion torus. The observation that $q \simeq 3$ fits the line profile would be satisfied, and the decline of line emission at $\xi \geq 1000$ might signal the point at which the flux is insufficient to ionize the disk atmosphere. The key parameter which determines the physical properties of the disk is probably $\dot{m}$. The close similarity between 3 C 390.3 and Arp 102B may indicate that they have roughly equal $\dot{m}$. The difference in absolute luminosity probably means that the mass of the black hole in Arp 102B is an order of magnitude smaller than that in 3C 390.3. The rarity of such objects might indicate that they occur only in a narrow range of $\dot{m}$. For example, if $\dot{m}$ is too large, a radiation torus may occupy the entire disk. If $\dot{m}$ is too small, an ion torus may fill the disk. Either of these tori are too hot to emit hydrogen lines. Only when an ion torus is limited to the inner disk, and illuminates the outer, thin disk, can double-peaked lines be observed. (See Begelman 1985 for a similar discussion of $\dot{m}$ in terms of unifying schemes for AGN types.) Taken together, the optical, infrared, and radio properties of 3C 390.3 and Arp 102B strongly support the theory of hot ion tori. 


\section{FUTURE WORK}

Although the line profile determines only the quantity $M / r$, one could measure the absolute radius of the disk, and hence the mass of the black hole, if inhomogeneities in the disk are observed to persist for at least one orbit. For an outer radius of $\xi=1000$ in Arp $102 \mathrm{~B}$, the rotation period is $3 M_{8}$ years. In fact, there have been slight variations in the line profile from year to year (Halpern and Filippenko 1988). Similarly, light travel times are of order $10^{6} M_{8} \mathrm{~s}$ across the diameter of the disk, so that variations in the line profile on a time scale of days are possible. Simultaneous continuum and line profile variations could provide independent confirmation of the rotating disk theory via the reverberation mapping technique (Blandford and McKee 1982), and also provide a measure of $M$. Fitting of Keplerian disk line profiles to more objects may result in the discovery of accretion disk components which make smaller and/or variable contributions to the broad line flux, as was claimed by Alloin, Boisson, and Pelat (1988) in the case of Akn 120. One would also hope to have a large enough sample of disks with measured inclination angles to evaluate possible viewing angle effects. If electron scattering is important, then the emission lines could be polarized. Finally, this work motivates a more quantitative calculation of the structure and emergent spectra of ion tori. Such an investigation has as its goal the prediction of the infrared and X-ray continua and their flux ratio, as well as the radiation spectrum of the illuminated outer disk.

We thank our collaborator, Alex Filippenko, for his invaluable help in obtaining and reducing the spectra used in this paper. This work was supported by NASA grant NAG 5-823, and is contribution number 359 of the Columbia Astrophysics Laboratory.

\section{REFERENCES}

Alloin, D., Boisson, C., and Pelat, D. 1988, Astr. Ap., in press.

Begelman, M. C. 1985, in Astrophysics of Active Galaxies and Quasi-Stellar Objects, ed. J. S. Miller (Mill Valley: University Science Books), p. 411.

Begelman, M. C. 1988, in Supermassive Black Holes, ed E. M. Kafatos (Cambridge: Cambridge University Press), p. 259.

Blandford, R. D., and McKee, C. F. 1982, Ap. J., 255, 419.

Chen, K., Halpern, J. P., and Filippenko, A. V. 1988, Ap. J., submitted (CHF).

Collin-Souffrin, S. 1987, Astr. Ap., 179, 60.

Halpern, J. P., and Filippenko, A. V. 1988, Nature, 331, 46.

Mardaljevic, J., Raine, D. J., and Walsh, D. 1988, Ap. Lett. Comm., 26, 357.

Miley, G., Neugebauer, G., Clegg, P. E., Harris, S., Rowan-Robinson, M., Soiffer, B. T., and Young, E. 1984, Ap.J. (Letters), 278, L79.

Oke, J. B. 1987, in Superluminal Radio Sources, eds. J. A. Zensus and T. J. Pearson (Cambridge: Cambridge University Press), p. 267.

Perez, E., Penston, M. V., Tadhunter, C., Mediavilla, E., and Moles, M. 1988, M.N.R.A.S., 230, 353.

Peterson, B. M., Meyers, K. A., Capriotti, E. R., Foltz, C. B., Wilkes, B. J., and Miller, H. R. 1985, Ap. J., 292, 164.

Rees, M. J., Begelman, M. C., Blandford, R. D., and Phinney, E. S. 1982, Nature, $295,17$.

Shakura, N. I., and Sunyaev, R. A. 1973, Astr. Ap., 24, 337.

Shields, G. A. and McKee, C. F. 1981, Ap. J. (Letters), 246, L57. 